\title{
ANL/PAY/CP-103562
}

\section{Recent Results of Radioactive Beam Experiments at ATLAS}

\author{
K. E. Rehm \\ Physics Division, \\ Argonne National Laboratory, \\ Argonne, IL 60439, USA
}

November 16, 2000

\begin{abstract}
Various beams of short- and long-lived radioactive nuclei have recently been produced at the ATLAS accelerator at Argonne National Laboratory, using either the so-called In-Flight or the Two-Accelerator method. With these beams many questions which are of interest in the areas of nuclear structure and nuclear astrophysics can be addressed. In this contribution first results from experiments with ${ }^{44} \mathrm{Ti}\left(\mathrm{T}_{1 / 2}=60 \mathrm{y}\right)$ and ${ }^{17} \mathrm{~F}\left(\mathrm{~T}_{1 / 2}=64 \mathrm{~s}\right)$ beams are discussed, which are of interest to the nucleosynthesis in supernovae and $\mathrm{X}$-ray bursts.
\end{abstract}

\section{Introduction}

Beams of unstable nuclei which are now available at various facilities worldwide have stimulated investigations of important questions in several areas of nuclear physics that could not be addressed previously. For example, weakly-bound nuclei at the neutron or proton drip line exhibit new structures, such as skins and halos, which have been shown to strongly influence reaction channels such as transfer or fusion. The effects of neutron-proton $\mathrm{T}=1$ pairing can best be studied in heavier $\mathrm{N}=\mathrm{Z}$ nuclei, which beyond ${ }^{40} \mathrm{Ca}$ are $\beta$-unstable. In nuclear astrophysics, it has been known for some time that a large fraction of the elements above $A \sim 20$ is produced in explosive nucleosynthesis where reactions occur on such a rapid time scale that unstable nuclei produced in these processes do not have time for $\beta$-decay, but rather continue to react with protons, neutrons or $\alpha$ particles which are available at the various astrophysical sites. While Nature has no difficulties producing these unstable, short-lived nuclei in the stellar furnaces, it was only during the last decade that some of these important reactions could be investigated in the laboratory. The intensities at first-generation facilities are still small, but the experiments have for the first time put some of the relevant astrophysical reaction rates on solid footing. In this contribution, experiments with ${ }^{17} \mathrm{~F}$ and ${ }^{44} \mathrm{Ti}$ beams, performed at the ATLAS accelerator at Argonne National Laboratory, will be discussed.

\section{Beam Production}

The majority of the radioactive beams are presently produced either via the isotope-separation-online (ISOL) technique or with the projectile-fragmentation method. In the ISOL approach, the radioactive 


\section{DISCLAIMER}

This report was prepared as an account of work sponsored by an agency of the United States Government. Neither the United States Government nor any agency thereof, nor any of their employees, make any warranty, express or implied, or assumes any legal liability or responsibility for the accuracy, completeness, or usefulness of any information, apparatus, product, or process disclosed, or represents that its use would not infringe privately owned rights. Reference herein to any specific commercial product, process, or service by trade name, trademark, manufacturer, or otherwise does not necessarily constitute or imply its endorsement, recommendation, or favoring by the United States Government or any agency thereof. The views and opinions of authors expressed herein do not necessarily state or reflect those of the United States Government or any agency thereof. 


\section{DISCLAIMER}

Portions of this document may be illegible in electronic image products. Images are produced from the best available original document. 
material is produced with a high-current driver accelerator or at a reactor. At sufficiently high temperatures some of the nuclei effuse out of the target material and are subsequently ionized and accelerated with a post accelerator.

In the fragmentation technique, a high-energy heavy-ion beam is fragmented in a thin target, and the fragments, emitted at forward angles with velocities close to the velocity of the incident beam are selected according to mass and nuclear charge in a fragment mass separator (a series of ion-optical elements) and then used directly in the experiment.

Beams produced with the ISOL method have characteristics (transverse and longitudinal emittance, beam spot size, energy variability) which are similar to those obtained at stable beam accelerators. For many nuclei, however, the effusion from the target is a relatively slow process which is also strongly dependent on the chemical properties of the element. Therefore mainly beams of long-lived elements, with high release efficiencies are available from this method. The complementary fragmentation technique, on the other hand, results in inferior beam qualities, but is very fast (typical flight times through the fragment separator are less than $1 \mu \mathrm{s}$ ) and independent of the chemical properties and, thus, short-lived nuclei are produced more efficiently by this method.

At the ATLAS accelerator at Argonne National Laboratory we have usec variations of the two techniques mentioned above for the production of several radioactive ion beams which are of interest to nuclear structure and astrophysics.

For longer-lived isotopes $\left(\mathrm{T}_{1 / 2} \geq 2 \mathrm{~h}\right)$ irradiated material is extracted from a production accelerator and converted into a suitable chemical form allowing the efficient production of positive or negative ions in an ion source. This allows to produce beams of nuclei which, because of their chemical properties, are difficult to extract using conventional ISOL techniques. Examples of beams produced by the twoaccelerator method at ATLAS are ${ }^{18} \mathrm{~F}$ and ${ }^{56} \mathrm{Ni}$.

For nuclei with shorter half-lifes, we have used the in-flight method[1] to produce beams of e.g. ${ }^{17} \mathrm{~F}\left(\mathrm{~T}_{1 / 2}=64 \mathrm{~s}\right)$ or ${ }^{25} \mathrm{Al}\left(\mathrm{T}_{1 / 2}=7.18 \mathrm{~s}\right)$. In this technique, a high-intensity beam of heavy ions from ATLAS with energies between $5-10 \mathrm{MeV} / \mathrm{u}$, incident on a gas cell filled with hydrogen, deuterium or ${ }^{3} \mathrm{He}$, generates via inverse $(\mathrm{p}, \mathrm{n}),(\mathrm{d}, \mathrm{n})$ or $\left({ }^{3} \mathrm{He}, \mathrm{n}\right)$ reactions secondary beams of unstable nuclei. These are separated from the primary beam and transported onto a secondary target that is to be studied. An example of this technique is the production of a ${ }^{17} \mathrm{~F}$ beam via the $\mathrm{d}\left({ }^{16} \mathrm{O},{ }^{17} \mathrm{~F}\right) \mathrm{n}$ reaction. In this case a few ppm of the primary ${ }^{16} \mathrm{O}$ beam particles are converted into ${ }^{17} \mathrm{~F}$ in the deuterium gas cell and transported through a $12 \mathrm{~m}$ long beam-line/separator system onto target. With a 100 pnA primary ${ }^{16} \mathrm{O}$ beam, a ${ }^{17} \mathrm{~F}$ intensity of $2 \times 10^{6}$ particles/s has been achieved. The experimental setup is shown schematically in Fig.1.

A superconducting bunching resonator, located $10 \mathrm{~m}$ upstream from the production target, provides a time focus of the primary beam at the gas cell, which minimizes the longitudinal emittance of the secondary beam. The ${ }^{17} \mathrm{~F}$ particles produced via the inverse $(\mathrm{d}, \mathrm{n})$ reaction are emitted within a cone with an opening angle of a few degrees. A superconducting solenoid located behind the target is used to capture the particles within this cone and to focus them through a $22^{\circ}$ bending magnet that separates the secondary ${ }^{17} \mathrm{~F}^{9+}$ particles from the primary ${ }^{16} \mathrm{O}^{8+}$ beam. The selection of the particles according to their magnetic rigidity results in a suppression of the primary beam by a factor of $\sim 3 \times 10^{-7}$. The debunching resonator located about $3 \mathrm{~m}$ behind the production target can be used to improve the energy spread of the secondary beam. By making use of the energy-time correlation and choosing the RF phase of this resonator appropriately, the energy resolution of the secondary beam has been improved by a factor of 3 (see Fig.2). The $400 \mathrm{keV}$ energy spread achieved for the ${ }^{17} \mathrm{~F}$ beam translates into a $23 \mathrm{keV}$ spread in the c.m. system for a study of the $\mathrm{p}\left({ }^{17} \mathrm{~F},{ }^{14} \mathrm{O}\right) \alpha$ system. The rebuncher behind the production target also allows to vary the energy of the secondary beams by about $\pm 5 \%$.

The beams produced with these two techniques at the ATLAS accelerator, including their energies RECEIVED 


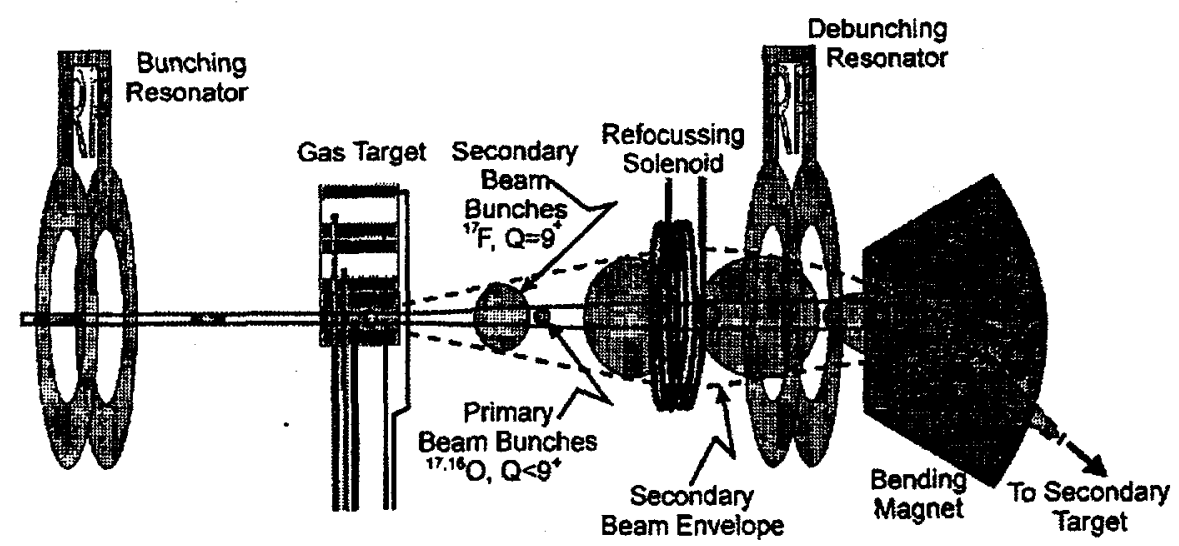

Figure 1: Schematic of the experimental setup used to produce short-lived radioactive beams via the in-flight technique.

and intensities, are summarized in Table.1.

\begin{tabular}{|c|c|c|c|c|c|}
\hline Beam & $\mathrm{T}_{1 / 2}$ & Production Method & $\begin{array}{c}\mathrm{I}_{\text {source }} \\
{\left[\mathrm{sec}^{-1}\right]}\end{array}$ & $\begin{array}{c}\mathrm{I}_{\text {target }} \\
{\left[\mathrm{sec}^{-1}\right]}\end{array}$ & $\begin{array}{c}\mathrm{E} / \mathrm{A} \\
{[\mathrm{MeV} / \mathrm{u}]}\end{array}$ \\
\hline${ }^{18} \mathrm{~F}$ & $110 \mathrm{~m}$ & two-accelerator & $5 \times 10^{7}$ & $3 \times 10^{6}$ & 0.6 \\
${ }^{56} \mathrm{Ni}$ & $6.1 \mathrm{~d}$ & two-accelerator & $2 \times 10^{7}$ & $6 \times 10^{4}$ & 5 \\
${ }^{56} \mathrm{Co}$ & $77 \mathrm{~d}$ & two-accelerator & $1 \times 10^{8}$ & $3 \times 10^{5}$ & 5 \\
${ }^{44} \mathrm{Ti}$ & $60 \mathrm{y}$ & two-accelerator & $2 \times 10^{7}$ & $5 \times 10^{5}$ & $2-7$ \\
${ }^{17} \mathrm{~F}$ & $65 \mathrm{~s}$ & in-flight & $5 \times 10^{7}$ & $3 \times 10^{6}$ & $3-6$ \\
${ }^{21} \mathrm{Na}$ & $22.5 \mathrm{~s}$ & in-flight & $2 \times 10^{7}$ & $5 \times 10^{5}$ & 5 \\
${ }^{25} \mathrm{Al}$ & $7.2 \mathrm{~s}$ & in-flight & $1 \times 10^{7}$ & $2 \times 10^{5}$ & 5 \\
\hline
\end{tabular}

Table 1: Radioactive ion beams produced at ATLAS.

A more detailed description of the two production methods can be found in Refs. $[1,2,3]$.

\section{Ion Source and Accelerator}

For the production of radioactive beams via the two-accelerator method (e.g. ${ }^{18} \mathrm{~F},{ }^{44} \mathrm{Ti},{ }^{56} \mathrm{Ni}$ ) a negative sputter source SNICS[4], dedicated to radioactive ion beams, has been installed at the Tandem accelerator which is one of the two injectors of the superconducting heavy-ion accelerator ATLAS. The 


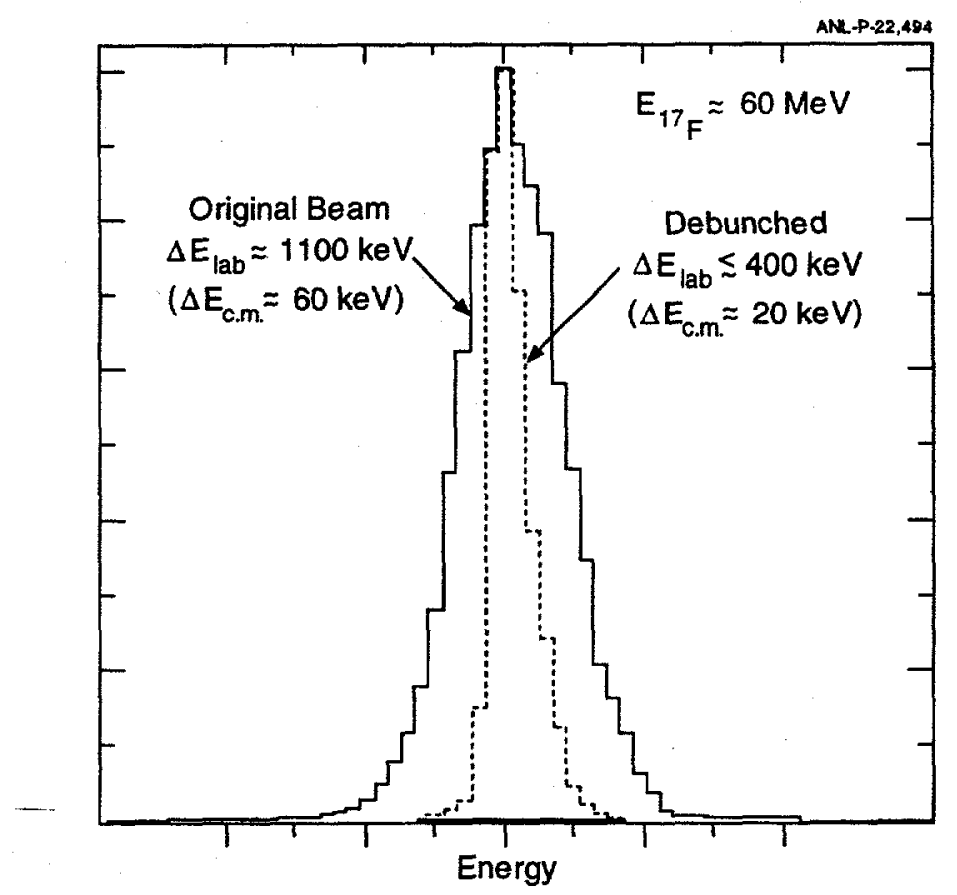

Figure 2: Effects of the debunching resonator on the energy distribution of a $60 \mathrm{MeV}$ beam of ${ }^{17} \mathrm{~F}$.

sputter source has quite large efficiencies for certain elements (e.g. $\sim 1 \%$ for fluorine) and it's compact geometry simplifies the decontamination after a run. For elements with small electron affinity (e.g. $\mathrm{Ti}^{-}$) molecules (e.g. $\mathrm{TiO}^{-}$) have been used.

The intensities of the radioactive beams extracted from the ion source are usually too weak to either stabilize the terminal voltage of the tandem accelerator or the timing of the bunched beam which is required for injection into the superconducting RF accelerator. For this reason a wedge-shaped Bicron Corporation plastic scintillator was mounted on a photomultiplier and installed in the vertical plane behind the tandem $90^{\circ}$ analyzing magnet. Timing signals from this scintillator were used to stabilize the time-of-arrival of the beam pulse by adjusting the phase of the pre-tandem buncher[5].

Another problem that had to be addressed was the tuning of the RF accelerator and the beam transport system. In some cases the stable isobar which is present in the source material can be used (e.g. ${ }^{18} \mathrm{O}$ for a ${ }^{18} \mathrm{~F}$ beam). In other cases, however, the intensity of this beam can overwhelm the detector system. For this reason the whole accelerator was tuned with a pilot beam of the same magnetic rigidity and velocity as the beam of interest. For $250 \mathrm{MeV}^{56} \mathrm{Ni}^{10+}$, a pilot beam of $125 \mathrm{MeV}^{28} \mathrm{Si}^{5+}$ was used, while for ${ }^{44} \mathrm{Ti}^{8+}$ the pilot beam was ${ }^{66} \mathrm{Zn}^{12+}$.

\section{Experimental Results}

In the next chapter results from two recent nuclear astrophysics experiments with ${ }^{17} \mathrm{~F}[6]$ and ${ }^{44} \mathrm{Ti}[7]$ beams are discussed in more detail. 


\subsection{Study of the ${ }^{17} \mathrm{~F}(\mathrm{p}, \alpha){ }^{14} \mathrm{O}$ Reaction}

Main-sequence stars with masses above $1.5 \mathrm{M}_{\odot}$ convert hydrogen into helium via the so-called CNO cycle which was first discussed by Bethe[8] and von Weizsäcker[9] more than 60 years ago. This reaction network, the catalytic fusion of four hydrogen atoms into helium, is shown by the double arrows in Fig.3. At temperatures typical of quiescent burning $\left(\mathrm{T} \sim 1-5 \times 10^{7} \mathrm{~K}\right)$, the average reaction times in this cycle are of the order of $10^{9}$ years and, thus, the unstable nuclei $\left({ }^{13} \mathrm{~N},{ }^{14} \mathrm{O}\right)$ produced in the reactions, have enough time to decay into their stable isobars. With increasing temperatures, however, the average reaction times decrease rapidly and the energy production in the CNO cycle will then be limited by the long $\beta$ decay half-life of ${ }^{13} \mathrm{~N}\left(\mathrm{~T}_{1 / 2}=10 \mathrm{~m}\right)$. This waiting point can be bypassed by the ${ }^{13} \mathrm{~N}(\mathrm{p}, \gamma)$ reaction leading to the nucleus ${ }^{14} \mathrm{O}$ which has an order of magnitude shorter half-life $\left(\mathrm{T}_{1 / 2}=70.6 \mathrm{~s}\right)$. At higher stellar temperatures occurring in explosive scenarios (e.g. X-ray bursts), the beta-decay of ${ }^{14} \mathrm{O}$ will become the waiting point limiting the energy production in the hot CNO cycle. This bottleneck is broken when, at higher temperatures, breakout via the ${ }^{14} \mathrm{O}(\alpha, \mathrm{p}){ }^{17} \mathrm{~F}$ reaction becomes possible leading to a more complex reaction network shown by the single arrows in Fig.3. This 'hot

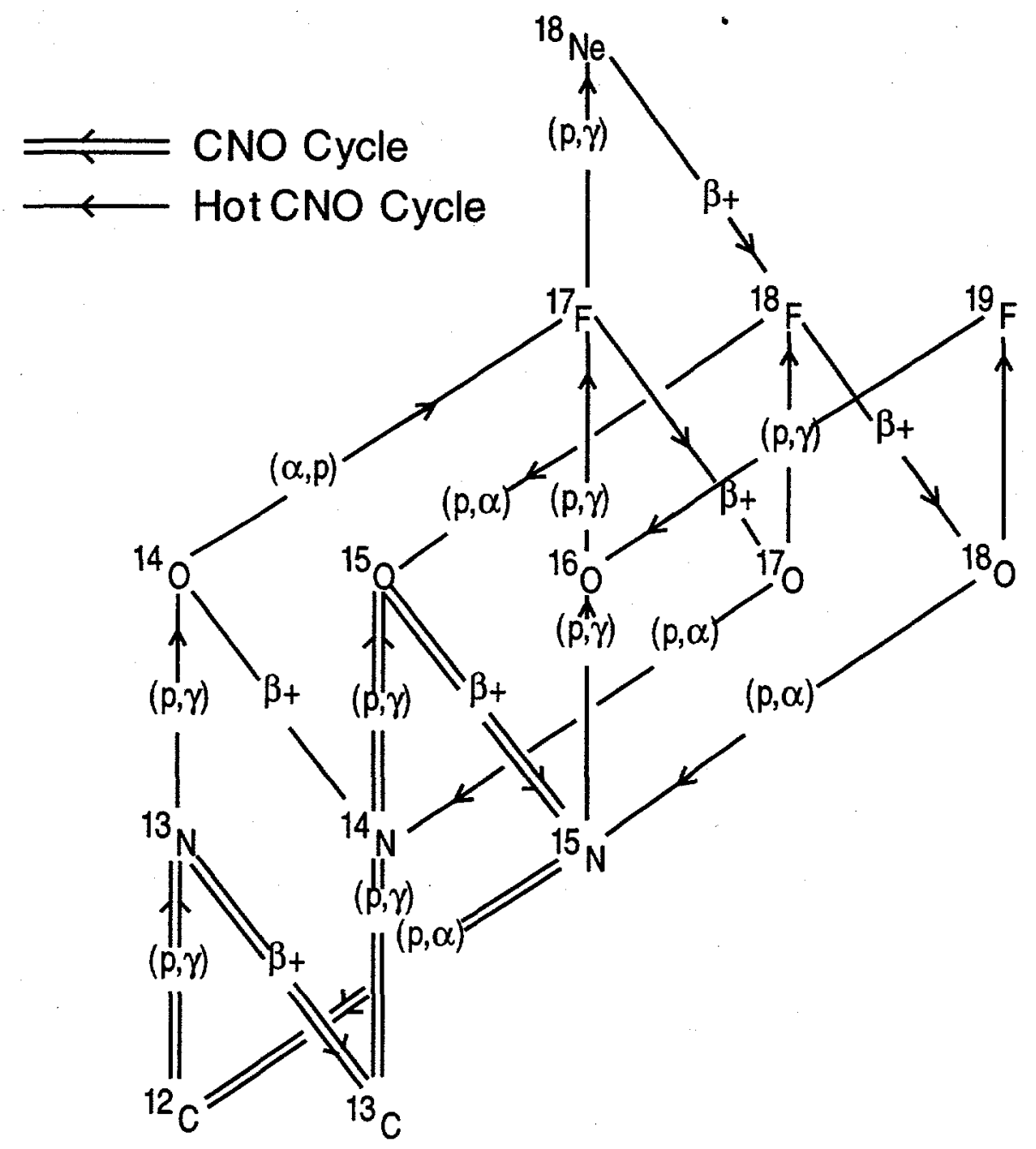

Figure 3: Reaction network for the CNO cycle (double arrows) and the 'hot CNO cycle (single arrows) 
- CNO cycle' involves reactions on unstable nuclei $\left({ }^{13} \mathrm{~N},{ }^{14} \mathrm{O},{ }^{17} \mathrm{~F},{ }^{18} \mathrm{~F}\right)$ which, in the past, could only be estimated on theoretical grounds. Only in the last 10 years have some of these reaction rates have been determined experimentally $[10,11,12,13,14,15]$

The second step in the hot CNO reaction network involves the ${ }^{14} \mathrm{O}(\alpha, \mathrm{p}){ }^{17} \mathrm{~F}$ reaction. A direct measurement of this reaction is difficult, since it requires a low-energy ${ }^{14} \mathrm{O}$ beam $\left(\mathrm{T}_{1 / 2}=70.6 \mathrm{~s}\right)$ as well as a He gas target. For this reason it is easier to study the properties of the natural parity states in the compound nucleus ${ }^{18} \mathrm{Ne}$ populated via the inverse reaction ${ }^{17} \mathrm{~F}(\mathrm{p}, \alpha){ }^{14} \mathrm{O}$ and use detailed balance to obtain the relevant widths $\Gamma_{p}$ and $\Gamma_{\alpha}$. A measurement of the inverse reaction ${ }^{17} \mathrm{~F}(\mathrm{p}, \alpha){ }^{14} \mathrm{O}$, however, does not provide any information about the proton width of the first excited $1 / 2^{+}$state in ${ }^{17} \mathrm{~F}$ that can be populated in explosive nucleosynthesis. The width of this state $\Gamma_{p^{\prime}}$ can be obtained from a measurement of the excitation function for inelastic scattering ${ }^{17} \mathrm{~F}\left(\mathrm{p}, \mathrm{p}^{\prime}\right) \mathrm{F}^{17}\left(1 / 2^{+}\right)$.

The ${ }^{17} \mathrm{~F}$ beam used in the experiments was obtained from the in-flight technique described above. $\mathrm{CH}_{2}$ targets with thicknesses of 100 and $500 \mu \mathrm{g} / \mathrm{cm}^{2}$ were used. The energy and the scattering angle of the (light and heavy) outgoing particles ( $\mathrm{p}, \alpha$ and ${ }^{14} \mathrm{O},{ }^{17} \mathrm{~F}$, respectively) were measured in coincidence using two position-sensitive double-sided annular silicon strip detectors. The measurement of the four quantities, $\theta_{\alpha}, \mathrm{E}_{\alpha}, \theta_{14} \mathrm{O}$ and $\mathrm{E}_{14 \mathrm{O}}$ allowed for a clean identification of the ${ }^{17} \mathrm{~F}(\mathrm{p}, \alpha){ }^{14} \mathrm{O}$ reaction with a detection efficiency of about $65 \%$. The measured cross sections are shown in Fig.4.

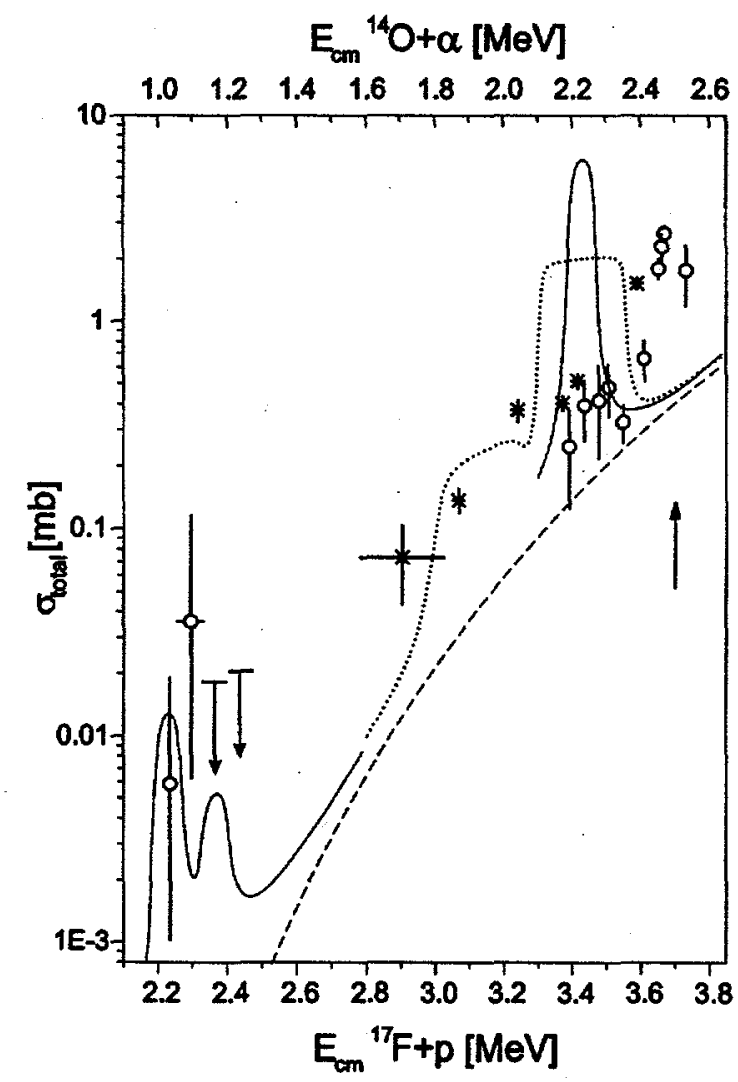

Figure 4: Cross sections measured for the ${ }^{17} \mathrm{~F}(\mathrm{p}, \alpha){ }^{14} \mathrm{O}$ reaction. The symbols are explained in the text. 
- The circles represent measurements with a thin $\left(100 \mu \mathrm{g} / \mathrm{cm}^{2}\right)$ target, while the crosses are obtained with a $500 \mu \mathrm{g} / \mathrm{cm}^{2}$ target. The horizontal bars, shown for one point only, indicate the energy interval covered by the target thickness. The dotted line is the expected thick target yield using parameters from Ref.[16], while the solid line is that for the thinner target. The dashed line represents the direct component estimated in Ref.[17]. The difference between the observed and the expected cross sections is due to incorrect spin-assignments for particle-unbound states in ${ }^{18} \mathrm{Ne}$ and to the omission of higher-lying states in Ref.[16].

From these experiments, the astrophysical reaction rate for the ${ }^{14} \mathrm{O}(\alpha, \mathrm{p}){ }^{17} \mathrm{~F}$ reaction in the tem-perature range $T_{9}=1-3$ is found to be about a factor of 2 smaller than previously assumed. However, these experiments did not yet take into account the question of the partial widths of proton decay to the excited $1 / 2^{+}$state in ${ }^{17} \mathrm{~F}$ which can be populated in the ${ }^{14} \mathrm{O}(\alpha, \mathrm{p}){ }^{17} \mathrm{~F}$ reaction. Preliminary results from a measurement of the ${ }^{17} \mathrm{~F}\left(\mathrm{p}, \mathrm{p}^{\prime}\right)^{17}$ reaction show that these transitions are generally smaller than the transition to the ground state[18].

\subsection{Study of the ${ }^{44} \mathrm{Ti}(\alpha, p){ }^{47} \mathrm{~V}$ Reaction}

Nuclei of ${ }^{44} \mathrm{Ti}$ are produced in the last stages of a supernova event in the so-called alpha-rich freezeout[19]. Because of the high energy release during a supernova explosion the nuclei in the shells outside the proto-neutron star (e.g. ${ }^{16} \mathrm{O},{ }^{28} \mathrm{Si}$..) are broken up into their constituents $(\mathrm{p}, \mathrm{n}, \alpha)$. During the subsequent expansion phase ' $\alpha$-nuclei', such as ${ }^{12} \mathrm{C},{ }^{16} \mathrm{O},{ }^{28} \mathrm{Si} . .$. are formed again, mainly through a series of $(\alpha, \gamma)$ reactions. The amount of a particular isotope generated during the $\alpha$-rich freeze-out in a supernova is governed by a subtle interplay between the nuclear reactions that produce it and those that destroy it. For ${ }^{44} \mathrm{Ti}$ the ${ }^{44} \mathrm{Ti}(\alpha, \mathrm{p})^{47} \mathrm{~V}$ reaction has been shown[20] to have the strongest impact: a small ${ }^{44} \mathrm{Ti}(\alpha, \mathrm{p})$ cross section will make ${ }^{44} \mathrm{Ti}$ a 'waiting point' in the $\alpha$-rich freeze-out, increasing the amount of ${ }^{44} \mathrm{Ti}$ produced, while a larger cross section results in an increased flow towards ${ }^{56} \mathrm{Ni}$, reducing the yield of ${ }^{44} \mathrm{Ti}$.

Because of its relatively long half-life of 60 years, ${ }^{44} \mathrm{Ti}$ provides an important tracer for young (T $\sim 600 \mathrm{y}$ ) supernovae remnants. The recent observation of $\gamma$ rays associated with the decay of ${ }^{44} \mathrm{Ti}$ from the Cassiopeia A [21] and the Vela [22] supernova remnants showed that the ${ }^{44} \mathrm{Ti}$ afterglow can indeed be used to locate individual supernovae. With the launch of the next-generation gamma-ray observatory INTEGRAL, scheduled for 2001, it can be expected that even more supernovae remnants will be discovered.

In order to study the amount of ${ }^{44} \mathrm{Ti}$ produced in a supernova explosion during the $\alpha$-rich freeze-out we have measured an excitation function of the ${ }^{44} \mathrm{Ti}(\alpha, \mathrm{p})^{47} \mathrm{~V}$ reaction. This experiment was performed with the recently developed ${ }^{44} \mathrm{Ti}$ beam at ATLAS. The material was produced via the ${ }^{45} \mathrm{Sc}(\mathrm{p}, 2 \mathrm{n})$ reaction using a $50 \mathrm{MeV}, 20 \mu \mathrm{A}$ proton beam from the injector of Argonne's Intense Pulsed Neutron Source. After a 70 hour long irradiation, $1.3 \mu \mathrm{g}$ of ${ }^{44} \mathrm{Ti}$ were produced which, after a cool-down of $\sim 1$ month, were chemically separated from the Sc host material and converted into $\mathrm{TiO}_{2}$ for the production of a $\mathrm{TiO}^{-}$beam in the negative ion sputter source.

The ${ }^{44} \mathrm{Ti}(\alpha, \mathrm{p}){ }^{47} \mathrm{~V}$ reaction was studied in inverse kinematics with a ${ }^{4} \mathrm{He}$ gas target and using the Fragment Mass Analyzer[23], a recoil mass separator dispersing the reaction products in its focal plane according to their $\mathrm{m} / \mathrm{q}$ ratios, for the separation of ${ }^{47} \mathrm{~V}$ from the primary mass 44 beam particles. To further separate the ${ }^{47} \mathrm{~V}$ particles from the scattered beam an ionization chamber (IC) and a Si detector were placed behind the parallel-grid-avalanche-counter (PGAC) at the focal plane of the fragment mass analyzer. The PGAC provided position $(\mathrm{m} / \mathrm{q})$ and timing signals, while the IC-Si detectors gave energy and $Z$ information. The He gas target consisted of a $2.2 \mathrm{~mm}$ long cell with two $1.3 \mathrm{mg} / \mathrm{cm}^{2}$ titanium metal windows, filled with 600 mbar of ${ }^{4} \mathrm{He}$ and cooled to $\mathrm{IN}_{2}$ temperature. The areal density of the 
target was $80 \mu \mathrm{g} / \mathrm{cm}^{2}$. Details of the experimental setup can be found in Ref.[7].

Figure 5 presents the measured cross section for the ${ }^{44} \mathrm{Ti}(\alpha, \mathrm{p})^{47} \mathrm{~V}$ reaction in comparison with results from the statistical model code SMOKER [24].

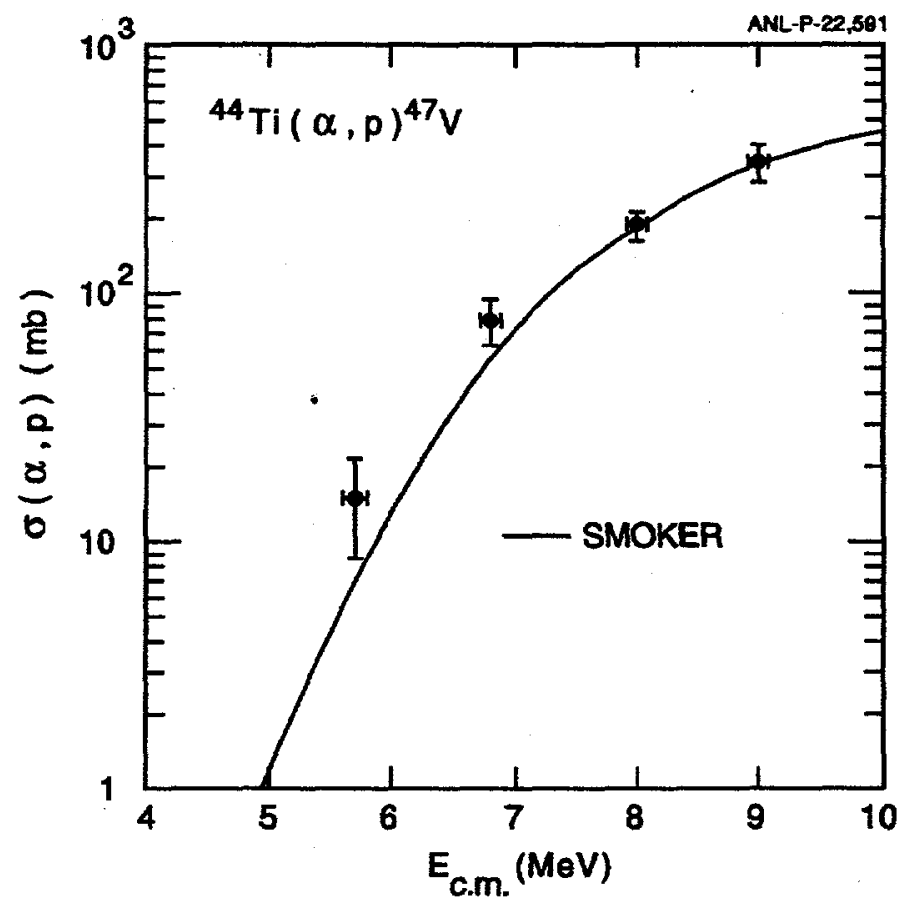

Figure 5: Experimental excitation function for the ${ }^{44} \mathrm{Ti}(\alpha, \mathrm{p})^{47} \mathrm{~V}$ reaction (solid points). The line corresponds to a calculation performed with the code SMOKER (see text for details).

While at the higher energies good agreement with the theoretical predictions is observed the falloff towards lower energies is slower than predicted resulting in cross sections that are about a factor of two larger than the SMOKER predictions. The important astrophysical quantity for calculating the processes in explosive nucleosynthesis is the astrophysical reaction rate, defined as the folding integral of the energy-dependent cross section with a Maxwellian velocity distribution[25]. The reaction rate for the ${ }^{44} \mathrm{Ti}(\alpha, \mathrm{p}){ }^{47} \mathrm{~V}$ reaction calculated from the experimental data is shown in Fig. 6 together with theoretical reaction rates taken from the literature[24,26,27,28] which are smaller than the experimental rate by factors of about $2-4$. This higher rate results in a reduction of the amount of ${ }^{44} \mathrm{Ti}$ produced in supernovae explosions. Using the calculations from Ref.[20] one obtains from these reaction rates a $25 \%$ decrease in the production yields of ${ }^{44} \mathrm{Ti}$ for a type-II supernova. For a measurement of the ${ }^{44} \mathrm{Ti}$ gamma-ray flux, this decrease in yield translates into a $15 \%$ larger distance or a 25 yr earlier occurrence of the supernova. However, it should be noted that changes in other reaction rates which have not been measured so far, could affect the ${ }^{44} \mathrm{Ti}$ yield as well. 


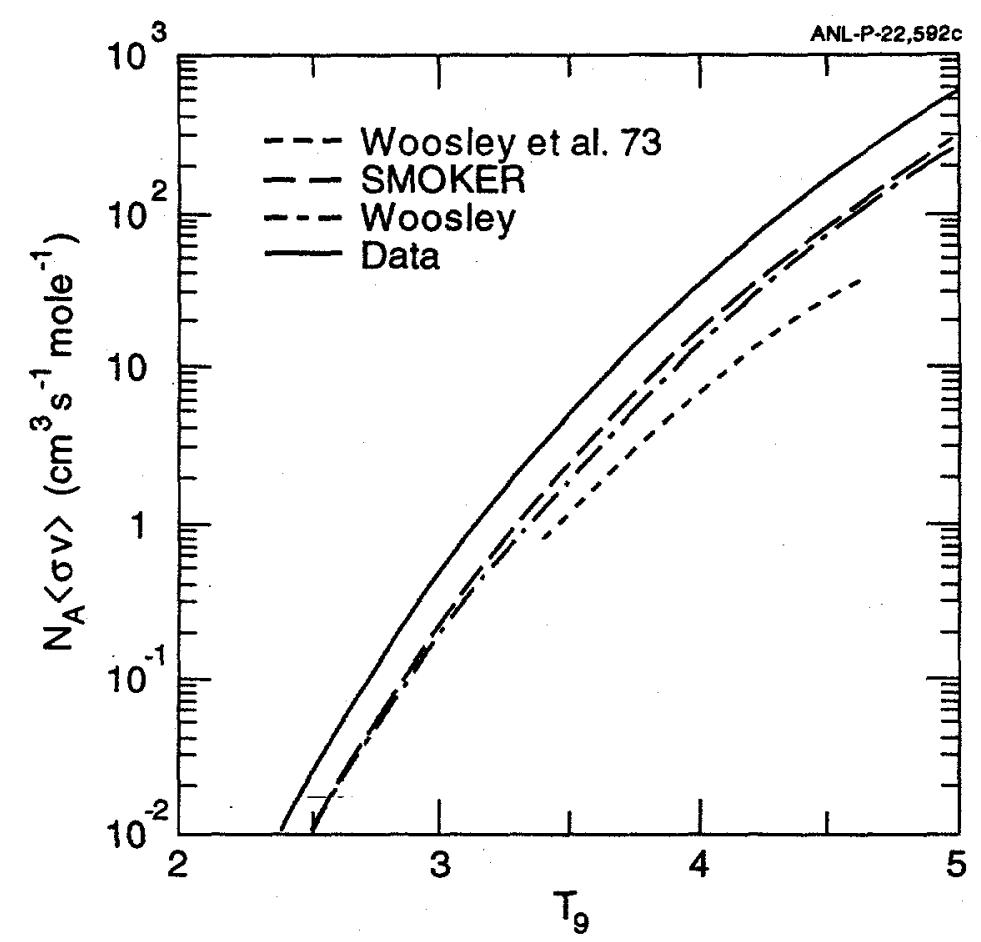

Figure 6: Astrophysical reaction rates for the ${ }^{44} \mathrm{Ti}(\alpha, \mathrm{p})^{47} \mathrm{~V}$ reaction. The solid line was obtained from the experimental data as described in the text. The dashed, dotted and dot-dashed lines correspond to theoretical rates taken from the literature.

\section{Conclusions}

Experiments with radioactive ion beams provide the opportunity to address many questions in nuclear structure and in nuclear astrophysics which have been unaccessible to experiments before. In nuclear astrophysics reactions with short-lived nuclei determine the time scale, the energy production and the nucleosynthesis in violent stellar explosions. The uncertainties in our understanding of these processes come from two sources, from an incomplete treatment of the complicated hydro-dynamical processes and from the use of theoretical reaction rates that need experimental confirmation. First results from experiments performed at existing radioactive beam facilities are now available for some of the reactions that play a role in the reaction networks in explosive nucleosynthesis. A full understanding of the nuclear physics occurring in these stellar processes, however, requires a next-generation facility with considerable higher beam intensities.

\section{Acknowledgment}

I want to thank all my collaborators from Refs.[6] and [7], especially B. Harss, C. L. Jiang and A. Sonzogni for their efforts in these experiments. This work was supported by the U. S. Department of Energy, Nuclear Physics Division, under Contract No. W-31-109-ENG-38, W-7405-Eng-48, DE-FG0298ER41086, DE-FG02-91ER-40609 and DE-AC05-96OR22464. 


\section{References}

[1] B. Harss et al., Rev. Sci. Instr. 71, 380 (2000)

[2] A. Roberts et al. Nucl. Instr. and Meth. B103, 523 (1995)

[3] K. E. Rehm et al., Nucl. Instr. and Meth. A449, 208 (2000)

[4] National Electrostatics Corporation, Graber Road, Box 310, Middleton, WI 53562

[5] G. P. Zinkann et al., $8^{\text {th }}$ International Conference on Heavy Ion Accelerator Technology, Argonne National Laboratory, Argonne, 1998, AIP Conference Proceedings Vol 473, 1999, p.279

[6] B. Harss et al., Phys. Rev. Lett. 82, 3964 (1999)

[7] A. A. Sonzogni et al., Phys. Rev. Lett. 84, 1651 (2000)

[8] H. A. Bethe, Phys. Rev. 103, 433 (1939)

[9] C. F. von Weizsäcker, Z. Phys. 39, 633 (1938)

[10] P. Decrock et al., Phys. Rev. Lett. 67, 808 (1991)

[11] T. Motobayashi et al., Phys. Lett. B264, 259 (1991)

[12] J. Kiener et al., Nucl. Phys. A552, 63 (1993)

[13] K. E. Rehm et al., Phys. Rev. C52, R460 (1995)

[14] R. Coszack et al., Phys. Lett. B353, 184 (1995)

[15] D. W. Bardayan et al., Phys. Rev. Lett 83, 45 (1999)

[16] K. I. Hahn et al., Phys. Rev. C94, 1999 (1996)

[17] C. Funck et al., Z. Phys. A332, 109 (1989)

[18] B. Harss et al., to be published.

[19] David Arnett, Supernovae and Nucleosynthesis (Princeton University Press, Princeton, NJ, 1996)

[20] L. S. The et al., Astrophys. J. 504, 500 (1998)

[21] A. F. Iyudin et al., Astron. Astrophys. 284, L1 (1994)

[22] A. F. Iyudin et al., Nature (London) 396, 142 (1998)

[23] C. N. Davids et al., Nucl. Instrum. Methods Sect. B70, 358 (1992)

[24] T. Rauscher et al., Phys. Rev. C56, 1613 (1997)

[25] C. E. Rolfs and W. S. Rodney, Cauldrons in the Cosmos (Chicago University Press, Chicago, IL 1988)

[26] S. Woosley et al., At. Data Nucl Data Tables 22, 371 (1978)

[27] S. Woosley et al., Astrophys. J. Suppl. 26, 231 (1973)

[28] J. W. Truran, Ap. and Space Sci. 11, 430 (1971) 